\title{
LOWER-LIMB ASYMMETRY IN BALANCE ABILITY OF YOUNG BADMINTON PLAYERS
}

\author{
Anna Manolova \\ National Sports Academy "Vassil Levski”"
}

\begin{abstract}
Based on the limited evidence available about balance performance among young badminton players, this study was aimed to establish the presence or absence of lower-limb balance asymmetry among 13-15 years old players.

The research was done among 40 young players (22 girls, 18 boys) from 3 Bulgarian clubs. Unipedal stance test and Y balance test were used to determine balance abilities. Paired sample T test and Wilcoxon signed-rank test were used to evaluate the data from the dominant and non-dominant leg.

The results from the two tests show that there is no statistically significant difference between the legs.

These results support the usefulness of performing bilateral balance exercises, so that the asymmetry that players are predisposed to develop could be reduced and the risk of lower extremity injuries would be decreased.
\end{abstract}

Key words: badminton, balance ability, leg asymmetry

\section{INTRODUCTION}

Badminton is considered as one of the most popular racket sports over the world. Badminton World Federation reports that there are around 150 million people playing badminton worldwide and more than 2000 participate in international competitions. Badminton players need to conduct various movement patterns during the game including specialized twists, jumps, footwork, and swings to strike the shuttlecock and keep it moving back and forth on the court. Thus, the game is characterized by a changing temporal structure with actions of short period and high or medium intensity coupled with a short resting times (Phomsoupha, Laffaye, 2015). Badminton requires specific physical conditions (motor and action controls); coordinative variables such as reaction time, foot stepping and static or dynamic balances. Therefore, badminton players need enough strength and a high level of balance during the rapid postural movements around the court (Hamed, Hassan, 2017).

Balance can be defined as "the ability to maintain the body's center of gravity within the limits of stability as determined by the base of support". Static postural control tries to maintain a base of support while minimizing movement of body segments and the center of mass. Dynamic balance involves the completion of a functional task with purposeful movements without compromising an established base of support (Yim-Chiplis, Talbot, 2000). Balance requires interaction of the neurologic, musculoskeletal, proprioceptive, vestibular and visual systems. Studies found that loss of balance during fast side-to-side movements may contribute to lower-limb injuries on the one hand, and on the other hand - that loss will influence performance levels, by decreasing them. Therefore, it is important to enhance player's balance ability. 
In 2012, the Games of XXX Olympiad (London Olympic) reported badminton to be one of the highest risky games for athletic injury (Engebretsen et al, 2013). Badminton match requires high intensity intermittent actions within short resting period. Fatigue may affect the neuromuscular control of lower limb, which increases the risk of injuries (Hiemstra, Lo, Fowler, 2001). A retrospective study among Malaysian badminton players reported about $60 \%$ of injuries happened when they were younger than 20 years and the most common injuries involved mainly the knees (Shariff, George, Ramlan, 2009). A study about the injuries of young European badminton players show a relatively high number of lower leg acute injuries in the examined badminton players as well as an asymmetry in the injured leg - they predominantly injure the leg on the side of the dominant hand, where the racquet is held (Petrinović, Bobic, Ciliga)

As one of racquet sport games badminton is unilateral and therefore structurally asymmetric sport. Functional asymmetries in the lower-limbs have been the subject of numerous investigations concerning many different contact, limited-contact and non-contact sports aimed at understanding the role of conditions in performance and in injury prevention. To return a shuttlecock falling near the net, badminton players lunge forward mainly by moving their dominant legs. This pattern of movement places a great burden on the muscles of the dominant leg. On the other hand, when the shuttlecock falls in the back section of the court, players jump off the dominant to the non-dominant leg to return it on some occasions and these movements greatly load the non-dominant leg (Masu, Muramatsu, Hayashi, 2014). There are several studies in which asymmetries in racquet sports were enquired, primary in tennis, but only few studies (Raschka, Schmidt, 2013; Petrinović, Štefan,
2015) in which morphological differences between dominant and non-dominant sides were determined among junior badminton players. We couldn't find evidence for exploration the presence/absence of balance asymmetry among 13-15 years old badminton players.

\section{Aim of the study}

The purpose of the study is to investigate lower-limb balance asymmetry among 13-15-year-old Bulgarian badminton players with the use of static and dynamic field tests. The application of field tests is important, so that they can be used by coaches from clubs, which cannot afford expensive equipment.

\section{Hypothesis}

Based on our knowledge about the game characteristics and our personal experience in terms of implementation of bilateral exercises during badminton training session, it was easy to formulate the following hypothesis: we assume that there will be no leg differences in balance performance, assessed by the static and the dynamic test. The occurrence of such asymmetry would mean wrong training protocols and would be of high informative value about the possible occurrence of injuries.

\section{METHODS}

\section{Participants}

Forty young badminton players aged between 13 and 15 years (boys $=18$, girls $=22$ ), participated in the study. They are ranked among the first fifteen of the national championships. All participants and their coaches provided informed consent. The players did not have any health problems or disability and had at least 2-year prior badminton experience.

\section{Protocol}

Participants performed two tests, evaluating dynamic balance (using the $\mathrm{Y}$ balance test) 
and static balance (using the Unipedal stance test). Before each test the participants were given practice trials to become familiar with the testing procedures - six times to minimize the learning effect for the $\mathrm{Y}$ balance test and two times for the Unipedal stance test. The leg on the side on which they gripped the racket was considered the dominant limb (D leg).

The players were given 5 minutes of slow running and 2-3 minutes for warm up of all body segments. Following a 3 minutes' rest period, participants began with the $\mathrm{Y}$ balance test - they performed three trials in each of the three directions, beginning with a right stance leg in the center of the grid. After another 5-minutes rest period, the test continued with a left stance leg.

Between the two balance tests, the participants received a 10-minute rest period. The Unipedal stance test was repeated three times with each leg, alternating the legs - one trial with the right, followed by a trial with the left leg. Five minutes of rest were allowed between each trial set to avoid fatigue.

For the proper conduct of the experiment, two investigators performed the testing - one dealing with the correction and observation of errors made by the competitor, and the other responsible for marking the reached distance at the Y balance test, stopping the watch at the Unipedal stance test, and recording the results from both tests.

\section{Procedures}

For the $\mathrm{Y}$ balance test participants stood on one leg on a center of Y - Junction shape surface. While maintaining single-leg stance, the player was asked to reach with the free limb in the anterior, posteromedial, and posterolateral directions in relation to the stance foot. The maximal reached distance was measured by marking the tape measure with erasable ink at the point where the most distal part of the foot reached. The trial was discarded and repeated if the player (1) failed to maintain unilateral stance, (2) lifted or moved the stance foot from the grid, (3) touched down with the reach foot, or (4) failed to return the reach foot to the starting position. The process was repeated while standing on the other leg. The greatest of 3 trials for each reached direction was used for analysis of the reached distance. As the reached distance is associated with limb length, it was normalized to limb length to allow more precise comparison between players. To express the reached distance as a percentage of limb length, the normalized value was calculated as reached distance divided by limb length then multiplied by 100 (Plisky et al., 2006).

For the Unipedal stance test the subjects were asked to stand barefoot on the limb of their choice, with the other limb raised so that the raised foot was near but not touching the ankle of their stance limb, arms crossed over the chest. When the participants closed their eyes, the investigator started the stopwatch. Time ended when the subject either: (1) used his arms (i.e., uncrossed arms), (2) used the raised foot (moved it toward or away from the standing limb or touched the floor), (3) moved the weight-bearing foot to maintain his balance (i.e., rotated foot on the ground), (4) a maximum of 45 seconds had elapsed, or (5) opened eyes. The procedure was repeated 3 times and each time was recorded on the data collection sheet. The average of the 3 trials was subjected to statistical processing (Springer et al., 2007).

\section{Statistical Analyses}

Data were analyzed with the use of SPSS Version 16.0 software. Descriptive statistics $($ Mean $\pm \mathrm{SD})$ were calculated for all variables. Data from Y balance test were compared with Paired Samples T test. Statistical significance 
was set at $\alpha \leq 0.05$ and $\mathrm{P}(\mathrm{t}) \geq 95 \%$. Data from Unipedal stance test were different from normally distributed, so we used Wilcoxon signed-rank test.

\section{RESULTS}

The descriptive statistic for the Unipedal stance test showed that the results could not be considered as normally distributed (Table 1).

Table 1. Results from the variation analysis of the Unipedal test's data

\begin{tabular}{c|c|c|c|c|c|c|c|c|c}
\hline Leg & $\mathbf{n}$ & $\mathbf{X} \mathbf{m i n}$ & $\mathbf{X} \mathbf{m a x}$ & $\mathbf{R}$ & $\overline{\mathbf{X}}$ & $\mathbf{S}$ & $\mathbf{V}$ & $\mathbf{A s}$ & $\mathbf{E x}$ \\
\hline Non-dominant & 40 & 3.22 & 36.93 & 33.71 & 12.65 & 8.24 & 65.14 & $1.71^{*}$ & $3.038^{*}$ \\
Dominant & 40 & 2.00 & 45.00 & 43.00 & 10.43 & 8.10 & 77.68 & $2.208^{*}$ & $7.43^{*}$ \\
\hline
\end{tabular}

* The empirical value is greater than the critical one

In Table 1 we can see that with both the dominant and non-dominant's data the empirical values of skewness (As) and kurtosis (Ex) are greater than the critical one (As = $1.71 / 2.208>\mathrm{As}_{0.05}=0.748 ; \mathrm{Ex}=3.038 / 7.43$ $\left.>\mathrm{Ex}_{0.05}=1.465\right)$. Checking the data, we ob- served some highly divergent values, which are actual measured results, and there is no reason to change them in the data set. These findings give us the right to use the Wilcoxon signed-rank test (T) to compare the samples (Table 2).

Table 2. Wilcoxon signed-rank test for the Unipedal stance test

\begin{tabular}{cccccc}
\hline & N & Sum of ranks & Mean rank & Z(T) & $\boldsymbol{\alpha}^{*}$ \\
\hline Positive Ranks & 27 & 523 & 19,37 & 1,519 & 0,129 \\
Negative Ranks & 13 & 297 & 22,85 & & \\
Ties & 0 & & & & \\
Total & 40 & & & & \\
\hline
\end{tabular}

${ }^{\star}$ Level of significance set at $\alpha \leq 0.05$

The sample size is 40 , so we can also use the $\mathrm{Z}$ evaluation of the test. Analyzing Table 2, we note that the empirical value of the test is $297\left(\mathrm{~T}_{\mathrm{emp}}=297\right)$ and the $\mathrm{Z}$ evaluation is 1.519 $\left(Z_{\text {emp }}=1.519\right)$. Comparing them to the critical values, we can come to the conclusion that there is no significant difference between the dominant and the non-dominant leg:

$$
\mathrm{T}_{\text {emp }}(297)>\mathrm{T}_{0,05}(264) ; \mathrm{Z}_{\text {emp }}(1.52)<
$$

$\mathrm{Z}_{0.05}(1,96)$; and $\alpha>0.05$.

The results from the variation analysis of the $\mathrm{Y}$ balance tests are presented in Table 3. We used the normalized values (dividing the reached distance by leg length and multiplying the score by one hundred), so that the comparison between the players would be more precise. 
Table 3. Descriptive statistic for the Y balance test

\begin{tabular}{ccccccccccc}
\hline Direction & Leg & $\mathbf{n}$ & $\mathbf{X}$ min & $\mathbf{X m a x}$ & $\mathbf{R}$ & $\mathbf{X}$ & $\mathbf{S}$ & $\mathbf{V}$ & $\mathbf{A s}$ & $\mathbf{E x}$ \\
\hline \multirow{2}{*}{ Anterior } & ND & 40 & 55.2 & 100 & 44.8 & 84.57 & 8.84 & 10.45 & -0.706 & 1.769 \\
& D & 40 & 57.3 & 109.5 & 52.2 & 86.36 & 10.70 & 12.39 & -0.028 & 0.556 \\
Post.medial & ND & 40 & 57.3 & 108.2 & 50.9 & 88.85 & 11.48 & 12.92 & -0.491 & 0.013 \\
& D & 40 & 53.1 & 112.9 & 59.8 & 88.73 & 12.83 & 14.46 & -0.405 & 0.319 \\
Post.lateral & ND & 40 & 54.2 & 114.1 & 59.9 & 87.76 & 12.00 & 13.68 & -0.425 & 0.402 \\
& D & 40 & 45.8 & 116.7 & 70.9 & 85.21 & 13.09 & 15.36 & -0.588 & 1.169 \\
\hline
\end{tabular}

*ND leg - Non-dominant leg

D leg-Dominant leg

Analyzing it we conclude that the data can the comparative analysis we can use the Pared be considered as normally distributed, so for Samples $\mathrm{T}$ test (Table 4).

Table 4. Paired Samples T test of the Y balance test

\begin{tabular}{cccccccc}
\hline \multirow{2}{*}{ Direction } & \multirow{2}{*}{$\mathbf{n}$} & ND leg & D leg & \multirow{2}{*}{ d } & \multirow{2}{*}{ Cohen d } & \multirow{2}{*}{ t value } & \multirow{2}{*}{$\mathbf{P}(\mathbf{t})$} \\
\cline { 3 - 6 } & & Mean \pm SD & Mean \pm SD & & & & \\
\hline Anterior & & $84,57 \pm 8,84$ & $86,36 \pm 10,70$ & -1.79 & 0,229 & 1,45 & 84,5 \\
Post. medial & 40 & $88,85 \pm 11,48$ & $88,73 \pm 12,83$ & 0.12 & 0,012 & 0,08 & 6,19 \\
Post. lateral & & $87,76 \pm 12,00$ & $85,21 \pm 13,09$ & 2.54 & 0,305 & 1,93 & 93,89 \\
\hline
\end{tabular}

${ }^{*}$ ND leg-Non-dominant leg

D leg - Dominant leg

Observing Table 2, we notice that the empirical values of the $\mathrm{T}$ test are smaller than the critical one: $\mathrm{t}_{\text {ant. }}=1.45, \mathrm{t}_{\text {p.m. }}=0.08, \mathrm{t}_{\text {p.l. }}=1.93$ $<\mathrm{t}_{0.05(\mathrm{df}=39)}=2.02$. From these results we can conclude that there is no statistically significant difference between the dominant and the non-dominant leg performance. To such conclusion also leads the $\mathrm{P}(\mathrm{t})$ value, which for all three directions is smaller than $95 \%$.

The effect size (Cohen d) for the anterior and the posterolateral direction is moderate $(0.2<$ Cohen $d<0.5)$ and for the posteromedial is small $(0.2>$ Cohen $d)$.

\section{DISCUSION}

The aim of this study was to investigate lower-limb balance asymmetry among 13-15-year-old Bulgarian badminton players. We couldn't find evidence for exploration the presence/absence of such asymmetry in badminton, investigated with field balance tests. Studying the literature, we found different points of view about symmetry between dominant and non-dominant leg.

Nadzalan et al. (2018) found no significant differences for all joint angles between dominant and non-dominant lower limb during step and jump forward lunge in badmin- 
ton. However, dominant lower limb was found to achieve faster ascend phase, descend phase and time to complete 1 repetition of lunge. Dominant limb was also showed to achieve greater step length. These conditions were applied to all the lunge protocols they conducted. These findings thus showed that imbalances existed between dominant and non-dominant limb during lunge movement (Nadzalan et al., 2018). The findings of this current study were in contrast to those found by study conducted among athletes practicing martial arts that found no significant difference of dominant and non-dominant lower limb kinematics when performing kicks (Falcó et al., 2009).

Teixeira et al. (2011) found symmetric balance stability between the right and left legs, analyzing the center of pressure in soccer players.

Aizawa et al. (2018) have examined limbdominance effects on the GRF during singleleg lateral jump-landings. Their study showed that the peak ground reaction force (pMGRF and M-pVGRF) is larger in the non-dominant leg compared to that in the dominant leg.

Petrinović, et al. (2015) investigated morphological status of elite European junior badminton players. The results of the study showed that statistical differences between opposite sides of the body of badminton players existed on forearm and upper leg circumferences.

The present study shows no statistically significant differences between the dominant and non-dominant leg in both tests. Although we found that at the posterolateral direction at the $\mathrm{Y}$ balance test there is an important difference. We can explain these results with the biomechanical characteristics of the sport. In their study Teixeira et al. (2011) indicate that in mobilization pedal skills both the right and left legs play an active role in task execution, with one leg producing the desired motion while the other leg stabilizes body balance. In another context, when stabilization is the main component of the action (e.g., keeping static balance on one foot), the supporting leg plays the main role while the contralateral leg remains motionless or is used to produce only auxiliary movements.

Although, this experiment supports our hypothesis, the data, to some extent, differ from our preliminary expectations. A number of surveys show that the $\mathrm{Y}$ balance test can be used as a predictor of lower-limb injuries. In their study Plisky et al. (2006) found that anterior right/left reached distance difference was greater than or equal to $4 \mathrm{~cm}$ and decreased normalized right anterior reached distance. They were significantly associated with lower extremity injury. Olmstead et al. (2003) found that players with chronic ankle instability had significantly decreased reach distances compared to the uninvolved limb and to the reach distances of healthy controls. The results of the anterior and the posterolateral direction put some concerns at our mind.

For the Unipedal stance test Springer et al. (2007) report 9.4 seconds as a mean time for the age group of 18-39. Both the dominant and the non-dominant mean time in our research are greater, but we have to consider that our participants are aged between 13 and 15 years. We should also consider applying the Unipedal stance test with open eyes, because this condition will be closer to the badminton game.

\section{CONCLUSION}

Badminton is a unilateral sport. Because of this, the movements of the dominant limb are more precise and quicker than those of the non-dominant limb, the players are predisposed to develop some imbalances. Asym- 
metry between sides of body can increase potential risk of injuries and negatively influence players' performances in the game. Although we didn't find statistically significant differences, some additional physical and balance exercises enabling symmetrical development, should be implemented in training. We also recommend additional observations and studies in this area, so as to avoid some negative consequences that a frequent and intensive badminton training could cause.

\section{REFERENCES}

Aizawa, J. et al. (2018). Limb-dominance and gender differencesin the ground reaction force during single-leglateral jump-landings, Journal of Physical Therapy Science, vol. 30, no. 3

Engebretsen L. et al (2013). Sports injuries and illnesses during the London Summer Olympic Games 2012. British Journal of Sports Medicine, vol. 47, pp. 407-414.

Falcó, C., Alvarez, O., Estevan, I., MolinaGarcia, J., Mugarra, F., \&Iradi, A. (2009). Kinetic and kinematic analysis of the dominant and non-dominant kicking leg in the taekwondo roundhouse kick, 27 International Conference on Biomechanics in Sports, 2009, Limerick, Ireland

Hamed, I., Hassan, I. (2017). The effect of core stability training on dynamic balance and smash stroke performance in badminton players, International Journal of Sports Science and Physical Education, vol. 2, no. 3, pp. 44-52

Hiemstra, L., Lo, I., Fowler, P. (2001). Effect of fatigue on knee proprioception: implications for dynamic stabilization, Journal of Orthopedic \& Sports Physical Therapy, vol. 31, pp.598-605

Masu, Y., Muramatsu, K., Hayashi, N. (2014). Characteristics of sway in the Center of Gravity of badminton players, Journal of
Physical Therapy Science, vol.26, pp. 16711674

Nadzalan, A. et al., (2018). Kinematics analysis of dominant and non-dominant lower limb during step and jump forward lunge in badminton, Journal of Fundamental and Applied Sciences, 10(3S), pp. 232-242

Olmsted, L. et al. (2003). Efficacy of the Star excursion balance tests in detecting reach deficits in subjects with chronic ankle instability, Journal of athletic training, vol.37, pp. 501-506.

Petrinović, L., Bobic, T., Ciliga, D. (2017). Knee injuries and overuse syndromes in European elite badminton players: is there a link between injury and training hours?, $8^{\text {th }}$ International Scientific Conference on Kinesiology, 2017, Opatija, Croatia

Petrinović, L., Štefan, L. and Munivrana, G. (2015). Some morphological differences between opposite sides of the body of elite European junior badminton players, Acta $\mathrm{Ki}$ nesiologica 9, 2: 67-71

Phomsoupha, M. and Laffaye, G. (2015). The science of badminton: game characteristics, anthropometry, physiology, visual fitness and biomechanics, Sports Med, vol. 45, no. 4, pp. 473-495

Plisky, Ph. et al. (2006). Star excursion balance test as a predictor of lower extremity injury in high school basketball players, Journal of Orthopedic \& Sports Physical Therapy, vol.36, is. 12

Raschka, Ch., Schmidt, K. (2013). Sports anthropological and somatotypical comparison between higher class male and female badminton and tennis players; Papers on Anthropology XXII, pp. 153-161

Shariff, A., George, J., Ramlan, A. (2009). Musculoskeletal injuries among Malaysian badminton players. Singapore Medical Journal, vol.50, pp. 1095-1097.

Springer B. et al. (2007), Normative val- 
ues for the Unipedal Stance test with Eyes Open and Closed; Journal of Geriatric Physical Therapy, vol.30, pp. 8-15

Teixeira, L. et al. (2011). Leg preference and interlateral asymmetry of balance stability in soccer players, Research Quarterly for
Exercise and Sport, vol. 82, no. 1, pp. 21-27

Yim-Chiplis, P., Talbot, L. (2000). Defining and measuring balance in adults, Biological research for nursing, vol. 1, no. 4, pp. 321331

\section{Corresponding author:}

Anna Manolova

Department "Football and tennis"

National Sports Academy "Vassil Levski"

Studentski grad, 21, Acad. Stefan Mladenov str.

Sofia 1700, Bulgaria

E-mail: karotoo@abv.bg 